
\title{
28 Research Square \\ Formulating the MCIDs Of the Quality of Life Scale for Patients With Systemic Lupus Erythematosus Based on ROC Curve
}

Honghong Xue

Huizhou Third People's Hospital

\section{Zheng Yang}

Guangdong Medical University

Chonghua Wan ( $\nabla$ wanchh@hotmail.com )

Guangdong Medical University https://orcid.org/0000-0002-7323-6837

\section{Wendy Wong}

Chinese University of Hong Kong Universities Service Center for China Studies: The Chinese University of Hong Kong

\section{Mingyang Chen}

Guangdong Medical University Liaobu Hospital

\section{Tong Xie}

Guangdong Medical College Zhanjiang Campus: Guangdong Medical University

\section{Research}

Keywords: Systemic lupus erythematosus, MCID value, ROC curve, sensitivity, specificity

Posted Date: August 30th, 2021

DOl: https://doi.org/10.21203/rs.3.rs-839218/v1

License: (9) This work is licensed under a Creative Commons Attribution 4.0 International License. Read Full License 


\section{Abstract}

Purpose: This study uses the ROC curve to formulate the MCID value of the Quality of Life Instruments for Chronic Diseases of Systemic lupus erythematosus (QLICD-SLE V2.0) scale.

Methods: Using the representative item "In general, would you say your health is" of the MOS item short from health survey(SF-36) as an anchor, the questionnaire of QLICD-SLE V2.0 and the anchor item were used to investigate the patients on the first day of hospitalization, and the day before the patient was discharged. 428 patients with lupus erythematosus (32 males, 395females; aged 13-76 years) from Zhanjiang Hospital and Kunming Medical University Hospital in China were participated in this one year longitudinal follow-up study. The ROC curve was constructed by using the classification based on the anchor item as the gold standard and the difference score of the scale as the test variable. The cut-off point corresponding to the maximum value of the Youden index in the ROC curve is taken as the minimum clinical importance difference (MCID) value of the QLICD-SLE (V2.0) scale.

Results: According to the ROC curve, the MCID of physical domain, psychological domain, social domain, QLICD-GM, specific domain and QLICD-SLE (V2.0) total scale are 8.3,2.3,0,2.7,9.2 and 3.2. Area under the ROC curve of QLICD-SLE (V2.0) is $0.898, P($ Area $=0.5)<0.001$, the sensitivity is $100 \%$, the specificity is $66.9 \%$.

Conclusion: This study recommended 3.2 as the MCID of QLICD-SLE (V2.0) scale. Compared with before treatments, if the scale score after treatments changes at least 3.2 points positively, then the treatment intervention can be considered as clinically significant. The ROC curve method is used to visualize the sensitivity and specificity. It is more convincing to use the corresponding cut-off point as the MCID.

\section{Background}

Systemic lupus erythematosus (SLE) is an autoimmune inflammatory connective tissue disease that affects multiple organs in young women [1].The highest incidence of SLE is in North America, with a prevalence rate of $241 / 100,000$ / year. Asian and Hispanic ethnic groups have middle incidence. SLE is a chronic progressive autoimmune disease that is easily recurrent and affects multiple systems. Studies found that the death of the SLE patients in Chinese population had higher concurrent infection and serious complications [2, 3]. Corticosteroids and immunosuppressants remain the main treatment strategies for SLE when disease progress. With the new development of stem cell transplantation or biological agents from cell or molecular biology in the treatment of the SLE, the clinical outcomes benchmark was catching attention [4].To evaluate the clinical outcomes of SLE along the recurrent episodes of this chronic disease, Quality of life (QOL) or patients reported outcomes (PRO) is often been used to evaluate the effect for medical decision. Both generic and disease specific QOL instruments are used to investigate the changes of SLE patients in clinical practice. Although generic QOL are commonly adopted for chronic diseases or tumors patients, the results were found to be less sensitive or irrelevant to patients [5]. Four disease specific QOL/PRO instruments are commonly used for SLE patients, that 
consisted of the SLE symptom checklist (SSC), the SLE Quality of Life Questionnaire (SLEQOL), Lupus Quality of Life (LupusQol) and the Quality of Life Instruments for Chronic Disease- Systemic lupus erythematosus (QLICD-SLE (V2.0)) [6, 7, 8]. The QLICD-SLE (V2.0) contains 47 items, of which 19 items focused on the symptoms of SLE while 28 items for reflection of the physical and mental health. This scale had good psychometric properties with retest reliability coefficient of 0.7 and split-half reliability coefficient is $0.9[9,10]$.

For evaluating of clinical outcomes, the interpretation of the Quality of life and clinical efficacy questionnaire score is particularly important [11]. However, the benchmark of QOL/PRO of calculation of scores that collected from subjective feeling somehow would still give statistical but not clinical meaningful changes. Therefore, the calculation of minimum clinical importance difference (MCID) by the anchor and distribution methods was highly recommended internationally [12]. In 1987, MCID is defined as the minimum questionnaire dimension score change recognized by the patient without considering side effects and costs, it is a bridge between statistics and clinical establishment relationship $[13,14]$. How to formulate a reasonable and credible MCID value is extremely critical of SLE for the new treatment will be important. Although the MCID in determining the medical decision or important for predicting the prognosis of SLE is important, the MCID value has not been established for the QLICD-SLE (V2.0). To calculate a reliable MCID value, this research uses the second edition revised on the basis of version 1 for investigation, and at the same time expands the source of sample size. The common MCID formulation methods, effective standard methods (anchor-based methods), distribution-based methods were commonly used according to the literature $[15,16,17,18,19,20,21]$. This study uses the recently commonly used ROC curve method to calculate the MCID value. An anchor item is used as the gold standard, and patients are grouped according to the results of the anchor item to construct ROC curve and calculate MCID of the QLICD-SLE (V2.0).

\section{Methods}

\section{Subjects' recruitment}

SLE patients, who were diagnosed and followed up during their consultation at the Hospital of Kunming Medical University and Zhanjiang hospital, were recruited. The recruitment was started from 2018.92019.5.

Subjects were recruited if they: (1) cognitive and physical capable of self-completion of questionnaire; (2) agreed to participate in the survey with the sought of informed consent from a trained research assistant.

Subjects were excluded according to the following exclusion criteria: (1) diagnosed of any mental illness who could not facilitate the self-completion of the questionnaire; (2) cognitive impairment, those who cannot fill in the questionnaire themselves; (3) refused to participate in the research.

\section{Survey methods}


This project was reviewed by the Ethics Committee of Guangdong Medical University and Kunming Medical University and found that the qualifications and experience of the project investigator met the requirements, and the research plan and the subject selection plan were in line with scientific and ethical principles. The participants were voluntary and the informed consent was obtained from all subjects. All methods are carried out in accordance with relevant guidelines and regulations.

With the consent being sought of the subjects, they completed the QLICD-SLE (V2.0) scale and the anchor item on the day of admission. Respondents answered completely according to their actual situation. The completed questionnaires were checked immediately by research assistant to ensure no missing value. If any missing items were found, subjects would be asked for completion for follow up assessment. The patients were asked to complete the same questionnaire before the discharge.

\section{Scale scoring}

QLICD-SLE (V2.0) questionnaire and the anchor item (in general, your health is: 1. Very good 2. Good; 3. Good; 4. Fair; 5. Poor) were used in this study for the SLE patients. The QLICD-SLE (V2.0) contains 47 items, of which 19 items focused on the symptoms of SLE while 28 items for reflection of the physical and mental health [22]. Each question adopts a 5-level scoring method, in which forward questions (that is, as the score increases, the higher the quality of life) is directly based on the original score to calculate $1,2,3,4,5$ points, and the reverse question (that is, as the score increases, the lower the quality of life), the score will be reversed, that is, 5, 4, 3, 2, 1, score. Add the scores of all the items included in each aspect to get the score of this aspect, and add the scores of all the items included in the scale to get the score of the total scale. In order to compare the scores of different aspects and different scales with each other, the original scores are converted into standard scores by the formula $S=(X-M i n) \times 100 / R, S$ is the standard score, and $\mathrm{X}$ is the rough score, Min is the minimum score of the dimension or total scale, $\mathrm{R}$ is the range of the dimension or total scale (that is, the difference between the maximum score and the minimum score)[10].

\section{Statistical methods}

The primary outcome of this study was to establish the MCID for the QLICD-SLE(V2.0). The specific steps for formulating MCID using ROC curve as recommended and adopted here [23]:

(1) Anchor-based grouping: If the patient had the same answer on the anchor item on the first day of hospitalization as the day before discharge, they were classified as "no change group". If the answer on the anchor item on the first day of hospitalization and the day before discharge of the patient had changed at least 1 point, they were classified as an "improvement group" or vice versa "deterioration group". Anchor groups were classified according to the changes in the options of anchor item before and after treatments, which are called anchor grouping.

(2) Study object: To formulate the MCID value which is defined as the minimum clinical significance change value, both patients in either the improvement or unchanged groups were included in the study by 
taking the minimum value of the unchanged group and its 95\% upper limit as the range of the MCID value.

(3) Construction of ROC curve: The anchor item was used as the gold standard of classification. The cutoff points in the range of values were used for the test classification, and the sensitivity and specificity of each cut-off point were calculated. Draw points with 1-specificity was used as the abscissa and sensitivity for the ordinate. The cut-off point when the Youden index in the ROC curve was the largest would be estimate as the MCID value.

Medcalc software is used for the statistical analysis of this study. According to the article of Hanley et al. [24], using Medcalc software to estimate the sample size, the minimum sample size required for calculation is 172 patients (an error $=0.1$; power $=90 \%$ ). Considering a $5 \%$ loss rate, this part of the study requires 181 patients at least.

\section{Results}

\section{Basic information of SLE patients}

428 patients participated in the first questionnaire survey (before treatments), 279 patients participated in two measurements before and after treatments. The basic information of 428 patients is shown in Table 1. The sample consisted of $51.9 \%$ of age $<30$ years old, $26.2 \%$ of age $30-39$. Most SLE patients were female (92.5\% vs 7.5\%) and married (62.9\%). The test-retest reliability of the QLICD-SLE(V2.0) is 0.93 . 
Table 1

Characteristics of the Sample $(n=428)$

\begin{tabular}{|c|c|c|c|c|c|}
\hline Characteristics & $\mathbf{N}$ & $\%$ & Characteristics & $\mathbf{N}$ & $\%$ \\
\hline Gender & & & Marital status & & \\
\hline Male & 32 & 7.5 & Married & 269 & 62.9 \\
\hline Female & 396 & 92.5 & Others & 159 & 37.1 \\
\hline Ethnic groups & & & Medical insurance & & \\
\hline Han & 398 & 93.0 & Self-paid & 193 & 45.1 \\
\hline \multirow[t]{2}{*}{ Others } & 30 & 7.0 & Partly public insurance & 131 & 30.6 \\
\hline & & & Public insurance & 104 & 24.3 \\
\hline Age & & & Occupation & & \\
\hline$<30$ & 222 & 51.9 & Factory Worker & 63 & 14.7 \\
\hline $30-39$ & 112 & 26.2 & Farmer & 108 & 25.2 \\
\hline $40-49$ & 64 & 14.9 & Teacher & 30 & 7.0 \\
\hline $50-59$ & 20 & 4.7 & Office-bearer & 15 & 3.5 \\
\hline$\geq 60$ & 10 & 2.3 & Others & 212 & 49.6 \\
\hline Income\# & & & Treatments & & \\
\hline Poor & 150 & 35.0 & Hormone joint immunosuppressant & 283 & 66.1 \\
\hline Fair & 269 & 62.9 & Hormone & 120 & 28.0 \\
\hline High & 8 & 1.9 & immunosuppressant & 2 & 0.5 \\
\hline Missing & 1 & 0.2 & other & 23 & 5.4 \\
\hline \multicolumn{6}{|l|}{ Education } \\
\hline Primary school & 200 & 46.8 & & & \\
\hline High school & 114 & 26.6 & & & \\
\hline College or higher & 113 & 26.4 & & & \\
\hline Missing & 1 & 0.2 & & & \\
\hline
\end{tabular}

The score of QLICD-SLE (V2.0) at baseline, follow-up assessment and the corresponding changes were shown in Table 2. It can be seen that the score of the QLICD-GM before treatments is $66.0 \pm 13.8$, and the 
score after treatments is $65.6 \pm 13.7$. QLICD-GM is a score that reflects the physiological, psychological, and social functions of a patient, with a wide coverage, so the score is greatly affected by many factors. The score of the specific module is $66.7 \pm 13.6$. before treatment and $67.1 \pm 13.4$. The score of the patientspecific module has improved after treatment, which means that the patient's disease has improved after treatment. The same goes for the total scale score.

Table 2

Standard score of QLICD-SLE(V2.0) $(n=279)$ and Correlations with Anchor

\begin{tabular}{|llllllll|}
\hline Domains & \multicolumn{2}{l}{$\begin{array}{l}\text { Before treatment } \\
\text { Mean SD }\end{array}$} & \multicolumn{2}{l}{\begin{tabular}{l}
\multicolumn{2}{l}{ After treatment } \\
Mean SD
\end{tabular}} & \multicolumn{2}{l}{$\begin{array}{l}\text { Differences } \\
\text { Mean SD }\end{array}$} & $\begin{array}{l}\text { Anchor-item } \\
\text { Correlation( } \boldsymbol{\eta})\end{array}$ \\
\hline Physical domain & 66.3 & 14.2 & 67.7 & 12.4 & 1.4 & 11.9 & 0.46 \\
\hline Psychological domain & 61.0 & 18.7 & 60.1 & 18.2 & -0.9 & 14.3 & 0.37 \\
\hline Social domain & 72.4 & 16.0 & 70.7 & 17.4 & -1.7 & 13.2 & 0.37 \\
\hline QLICD-GM & 66.0 & 13.8 & 65.6 & 13.7 & -0.4 & 10.4 & 0.47 \\
\hline Specific domain & 66.7 & 13.6 & 67.1 & 13.4 & 1.6 & 12.1 & 0.27 \\
\hline QLICD-SLE & 66.3 & 14.2 & 67.7 & 12.4 & 0.4 & 9.3 & 0.43 \\
\hline
\end{tabular}

The correlation coefficient between the anchor item and the QLICD-SLE(V2.0) scale is 0.43 , and the correlation coefficient with the physiological module, psychological module, social function module, and specific module for systemic lupus erythematosus are $0.46,0.37,0.37,0.27$, respectively.

\section{Group based on the anchor item}

In this study, the score of the patient anchor item and the score of the scale, the correlation coefficient is 0.43 . It is generally considered that the correlation is greater than 0.3 . Depending on the changes in the scores of the anchor items before and after treatment, the patients were divided into 3 groups.56 of the 279 patients, the anchor item score after treatment minus the anchor item score before treatment is greater than 0,Therefore, these patients were included in Improvement group and the mean value of the score difference of the QLICD-SLE (V2.0) is $4.7 \pm 10.5 .169$ of the 279 patients, the anchor item score after treatment minus the anchor item score before treatment is 0 .Therefore, these patients were included in No change group and the mean value of the score difference of the QLICD-SLE (V2.0) is 0.3 \pm 8.1.54 patients had a negative change. The mean value of the score difference of the QLICD-SLE (V2.0) is $-3.4 \pm 10.0$.The score information of different modules is shown in Table 3. 
Table 3

Difference in scores of scales under anchor grouping $(n=279)$

\begin{tabular}{|lllllll|}
\hline Domains & Anchor-based grouping & $\mathbf{N}$ & Minimum & Maximum & Mean & SD \\
\hline Physical domain & Improvement group & 56 & -22.2 & 47.2 & 5.5 & 14.1 \\
\cline { 2 - 7 } & No change group & 169 & -27.8 & 36.1 & 1.5 & 10.7 \\
\cline { 2 - 7 } & Worsening group & 54 & -30.6 & 33.3 & -3.3 & 11.8 \\
\hline Psychological domain & Improvement group & 56 & -34.1 & 50.0 & 4.0 & 15.4 \\
\cline { 2 - 7 } & No change group & 169 & -50.0 & 50.0 & -1.4 & 14.0 \\
\cline { 2 - 7 } & Worsening group & 54 & -40.9 & 20.5 & -4.3 & 13.3 \\
\hline Social domain & Improvement group & 56 & -28.1 & 25.0 & 1.3 & 10.2 \\
\cline { 2 - 7 } & No change group & 169 & -53.1 & 65.6 & -1.7 & 14.4 \\
\cline { 2 - 7 } & Worsening group & 54 & -34.4 & 18.8 & -4.9 & 11.6 \\
\hline QLICD-GM & Improvement group & 56 & -24.1 & 36.6 & 3.7 & 10.9 \\
\cline { 2 - 7 } & No change group & 169 & -38.4 & 29.5 & -0.6 & 9.9 \\
\cline { 2 - 7 } & Worsening group & 54 & -27.7 & 14.3 & -4.1 & 9.7 \\
\hline Specific domain & Improvement group & 56 & -17.1 & 48.7 & 6.0 & 13.6 \\
\cline { 2 - 6 } & No change group & 169 & -38.2 & 32.9 & 1.5 & 10.1 \\
\hline QLICD-SLE & Worsening group & 54 & -34.2 & 51.3 & -2.4 & 14.8 \\
\hline & Improvement group & 56 & -17.6 & 41.5 & 4.7 & 10.5 \\
\cline { 2 - 6 } & No change group & 169 & -27.7 & 17.6 & 0.3 & 8.1 \\
\cline { 2 - 6 } & Worsening group & 54 & -21.8 & 29.3 & -3.4 & 10.0 \\
\hline
\end{tabular}

\section{MCID values estimate by the ROC for the QLICD-SLE (V2.0)}

The MCID of physical domain, psychological domain, social domain, QLICD-GM, specific domain and QLICD-SLE (V2.0) are 8.3,2.3,0,2.7,9.2 and 3.2, respectively. The area under the curve (AUC) of the QLICDSLE (V2.0) are greater than 0.8 and the $p<0.001$. The cut-off point is 3.2 of QLICD-SLE (V2.0)(sensitivity = $100 \%$, specificity $=66.9 \%$ ). In other words, before and after treatment, the QLICD-SLE (V2.0) score should increase by at least 3.2 points before the treatment is considered effective. If it is only for the Specific domain of patients with systemic lupus erythematosus, the score must be increased by at least 9.2 points(sensitivity $=75.0 \%$, specificity $=82.8 \%$ ) before clinical treatment is considered meaningful. The results of MCID values corresponding to different modules in the scale are shown in Table 4. The ROC curves of the specific module and the entire scale are shown in Fig. 1 and Fig. 2. 
Table 4

Roc curve analysis results $(n=181)$

\begin{tabular}{|c|c|c|c|c|c|c|}
\hline \multirow[t]{2}{*}{ Indicators } & \multicolumn{6}{|l|}{ value } \\
\hline & $\begin{array}{l}\text { Physical } \\
\text { domain }\end{array}$ & $\begin{array}{l}\text { Psychological } \\
\text { domain }\end{array}$ & $\begin{array}{l}\text { Social } \\
\text { domain }\end{array}$ & $\begin{array}{l}\text { QLICD- } \\
\text { GM }\end{array}$ & $\begin{array}{l}\text { Specific } \\
\text { domain }\end{array}$ & $\begin{array}{l}\text { QLICD- } \\
\text { SLE(V2.0) }\end{array}$ \\
\hline $\begin{array}{l}\text { Area under the ROC } \\
\text { curve (AUC) }\end{array}$ & 0.822 & 0.826 & 0.820 & 0.886 & 0.843 & 0.898 \\
\hline $\begin{array}{l}\text { Significance level P } \\
(\text { Area }=0.5)\end{array}$ & $<0.001$ & $<0.001$ & $<0.001$ & $<.001$ & $<0.001$ & $<0.001$ \\
\hline Youden index J & 0.489 & 0.580 & 0.556 & 0.680 & 0.578 & 0.669 \\
\hline $\begin{array}{l}\text { Associated } \\
\text { criterion(MCID) }\end{array}$ & 8.3 & 2.3 & 0 & 2.7 & 9.2 & 3.2 \\
\hline Sensitivity & $66.7 \%$ & $100 \%$ & $91.7 \%$ & $100 \%$ & $75.0 \%$ & $100 \%$ \\
\hline Specificity & $82.3 \%$ & $57.9 \%$ & $63.9 \%$ & $68.1 \%$ & $82.8 \%$ & $66.9 \%$ \\
\hline
\end{tabular}

\section{Discussions}

The prevalence of SLE in my country is higher than that in Western countries, and the mortality rate in the past is very high. Nowadays, major breakthroughs have been made in treatment. The 10-year survival rate is greater than $80 \%$, but there is no cure, and patients need long-term medication. Long-term medication and prolonged illness repeatedly cause serious ideological and economic burdens to patients. How to evaluate the changes in the physical and psychological health of SLE patients is particularly important. Foreign countries have developed universal scales and specific scales for SLE patients, and they have been widely used. However, due to differences in cultural ideas and diets at home and abroad, the direct use of foreign scales cannot fully reflect the conditions of patients in my country.

In China, there are few reports on the development of quality of life scales for patients with systemic lupus erythematosus. The research team developed the first version of the Quality of Life Instruments for Chronic Disease- Systemic lupus erythematosus (QLICD-SLE (V1.0)) during 2003-2006, referring to the contents of foreign scales and compares them with the clinical practice of systemic lupus erythematosus. The second version of the system of Quality of Life Instruments for Chronic Disease QLICD has been developed since 2008, and the QLICD-SLE (V2.0) was formed in 2017[25]. At that time, the MCID value is not established for the systemic lupus erythematosus scale.

Therefore, in this study, the MCID value was established by the roc curve method, which further made up for the shortcomings in the actual application of the scale, and facilitated the clinical evaluation of the patient's treatment effect through the change of the scale score.

There are four common methods for calculating MCID values, such as anchor-based method, distributionbased method, etc. In recent years, Due to the shortcomings of traditional methods, Roc curve method, 
multiple regression method, etc. are proposed. With the combination of illustrating the sensitivity and specificity in the same graph, this can facilitate the statistically interpretation with high accuracy [26, 27]. The anchor-based MCID distribution method were recommended for application in the ROC curve to formulate the MCID in other important PRO instruments such as J.J. Wu et al use ROC curve calculating MCID for work productivity and activity impairment ( WPAI ) questionnaire [28]. In the ROC curve, the cutoff point corresponding to the maximum Youden index has relatively high sensitivity and specificity. Compared with the traditional anchor method and distribution method for formulating MCID values, the ROC curve-based method effectively combines the advantages of the anchor method and the distribution method. Different cut-off values to calculate a range of sensitivity and specificity according to the patients' respondent in projection of the increments of outcomes is the core of the anchor based methods.

ROC curve-based method takes into account measurement errors and makes full use of the sample size as much as possible. It is believed that the correlation coefficient between the scale score and the anchor item score is greater than 0.3 , and the sufficiency of the anchor is strong [29]. In this study, the correlation coefficient between the score of the anchor item and the QLICD-SLE (V2.0) scale before the treatment is 0.43. The anchor item is used as a comprehensive indicator to reflect the overall health of patients with systemic lupus erythematosus. To formulate the minimum clinically significant improvement difference of the QLICD-SLE (V2.0) scale, Patients were divided into three different groups according to the changes in the response of the anchor items before and after treatment.

According to Table 1, the mean value for different groups of patients are: +4.7 (improved group) >+ 0.30 (no change group) $>-3.4$ (deteriorated group). It implied the grouping of patients based on anchor item was feasible. For easy reference, the negative items in the scale were transformed positively, making the scale score more easily interpreted with the patient's health status. If the treatment is clinically meaningful to the patient, the patient's health will also be improved. After the treatment, the patient's score on the QLICD-SLE (V2.0) scale should be higher than before the treatment, that is, after the treatment. The scale's standardized score minus before treatment should be positive. However, in practical applications, the difference between the post-treatment and pre-treatment scale scores is positive or negative. In this study, the difference was used as the test variable, and the classification based on the anchor item was used as the gold standard to construct the ROC curve.

The MCID of QLICD-SLE (V2.0) is 3.2. The patient's scale score after treatment is at least 3.2 points higher than that before treatment, and the clinical treatment is considered meaningful. Through MCID determination, it is possible to further explore the different treatment methods, which is more effective and other research.

The samples of this study mainly come from the southern cities of Guangdong and Yunnan. The economic situation in southern China is relatively good, and the population flow is relatively large. Selecting patients from the above areas for investigation can reflect the status of patients with lupus erythematosus in the Chinese system to a certain extent. Two independent sample t-tests were used to 
analyze the scores of patients in Guangdong and Yunnan. The results showed that there is no statistical difference in the scores of patients in the two regions before treatment $(t=1.600, p=0.111)$. Considering a $5 \%$ loss rate, this part of the study requires 181 patients at least. In fact, the results of this survey are that 428 patients participated in the questionnaire survey.

To sum up, formulating MCID value based on ROC curve as a new method has both advantages and disadvantages. The ROC curve intuitively combines sensitivity with specificity and accurately reflects the specificity and sensitivity of an analytical method. However, in the formulation of MCID, an appropriate anchor needs to be selected as the classification gold standard. In practice, it is difficult to find a very appropriate anchor and different anchors will also produce different results. Some literatures [30] pointed out that using the data of the entire cohort to construct the ROC curve, the accuracy of formulating the MCID is higher. The MCID value of the QLICD-SLE (V2.0) scale developed in this study is 3.2, which can assist in determining whether the treatment has a clinical effect on SLE patients. However, due to the number of study subjects and the limitations of the survey points, the effectiveness of its promotion and use needs further verification.

\section{Conclusion}

The formulation of the scale MCID has always been the main concern of the questionnaire application. Existing methods for formulating MCID values, such as the anchor-based method and the distributionbased method, have advantages and disadvantages. Today, the ROC curve method avoids the situation of positive and negative offsets, but some patients will be lost due to the inclusion principle and require larger data support. Although imperfect, it is better than existing methods to a certain extent. The shortcomings of the ROC curve in formulating the MCID value are shown in the following aspects: (1) How to define the minimum change interval, whether to change one point or more points. (2) One of the keys to formulating MCID for ROC curve is the selection of an appropriate anchor, with one item as the anchor and multiple items as the anchor? This does not have an accurate conclusion. Therefore, the formulation of the MCID value still needs to refer to the values of various methods for comprehensive evaluation.

\section{Abbreviations}

QOL: Quality of life

QLICD-SLE (V2.0): Quality of Life Instruments for Chronic Diseases of Systemic lupus erythematosus

SF-36: the MOS item short from health survey

MCID: minimal clinically important differences

\section{Declarations}




\section{Ethics approval and consent to participate}

The study protocol and the informed consent form were approved by the IRB (institutional review board) of Guangdong Medical university (PJ2013037) and Kunming Medical University (30860248). The respondents were voluntary and provided written consent for participation.

\section{Consent for publication}

NA/Not Applicable.

\section{Availability of data and materials}

The data (two formats: SPSS and Excel) can be available by request from Prof. Chonghua Wan (Email: wanchh.hotmail.com).

\section{Competing interests}

The authors declare that they have no competing interests.

\section{Acknowledgement}

The authors would like to thank the affiliated hospital of Guangdong Medical University and the First Affiliated Hospital of Hospital of Kunming Medical University for their support of this study and for their active cooperation.

\section{Funding}

Funded by the National Natural Science Foundation of China (71373058) and the Guangdong Science and Technology Program (2013B021800074)

\section{Authors' contributions}

$\mathrm{WCH}$ and $\mathrm{YZ}$ designed the study. $\mathrm{CMY}, \mathrm{XT}$ performed the data collection. $\mathrm{WCH}, \mathrm{XHH}$ performed data analyses, and all authors contributed to interpreting the data. $\mathrm{WCH}, \mathrm{XHH}$ and WW wrote the first draft, which was critically revised by all others. All authors have read and approved the final manuscript.

\section{References}

1. Ding FM. Cohort study and preliminary study on mechanism of systemic lupus erythematosus with early onset atherosclerosis. Peking Union Medical College, 2016.

2. You Y. Research progress of systemic lupus erythematosus in 2017. Dermatology and Venereology.2018;40 (02): 176-7.

3. Dong GF, Ye RG, Zhang X, Chen WL. Epidemiological study on concurrent infections of systemic lupus erythematosus .Chinese Journal of Nosocomiology.2004;(07): 39-42. 
4. Doward LC, Whalley D, Dewar AL, McKenna SP, Tennant A, Emery P. The development of the SLE-QoL: a quality of life instrument specific to systemic lupus erythematosus. Poster presented at the 6th Annual Conference of the International Society for Quality of Life Research (ISOQOL); November 1999. Barcelona, Spain. Qual Life Res. 1999 ; 8(7):609.

5. Fan JF,Shen Y.Efficacy and evaluation of a new treatment method for systemic lupus erythematosus .Chinese Journal of Practical Pediatrics.2012;27 (09): 663-6.

6. Grootscholten C, Ligtenberg G, Derksen RH, Schreurs KM, de Glas-Vos JW, Hagen EC. Health-related quality of life in patients with systemic lupus erythematosus: development and validation of a lupus specific symptom checklist. Qual Life Res. 2003;12(6):635-44. DOI:10.1023/a:1025176407776

7. Leong KP, Kong KO, Thong BY, Koh ET, Lian TY, Teh CL,et al. Development and preliminary validation of a systemic lupus erythematosus-specific quality of lifeinstrument(SLEQOL) .Rheumatology(Oxford).2005;44: 1267-76.DOI: 10.1093/rheumatology/keh605

8. McElhone K, Abbott J, Shelmerdine J, Bruce IN, Ahmad Y, Gordon C,et al. Development and validation of a disease-specific health-related quality of life measure, the LupusQol, for adults with systemic lupus erythematosus. Arthritis Rheum. 2007;57(6):972-9.DOI: 10.1002/art.22881

9. Yu YL, Lu ZP, Wan CH, Wang GH, Xu J, Xu CZ, Jia S. Reactivity and feasibility evaluation of systemic lupus erythematosus quality of life scale. Chinese Journal of Behavioral Medicine and Brain Science.2017;26 (5): 471-4.

10. Yu YL, Lu ZP, Wan CH,Wang GH, Xu J, Xu CZ, Jia S. Development of systemic lupus erythematosus scale for patients with chronic diseases and its reliability and validity. Chinese Journal of Disease Control \& Prevention.2013;(11): 997-1001.

11. Hu GQ,Huang QF,Huang ZN,Sun ZQ Methods for determining the minimum clinical significance change value in clinical research.Journal of Central South University (Medical Edition),2009;34(11):1058-62.

12. Kathleen W Wyrwich, Monika Bullinger, Neil Aaronson, Ron D Hays, Donald L Patrick, Tara Symonds, Clinical Significance Consensus Meeting Group. Estimating clinically significant differences in quality of life outcomes. Qual Life Res.2005;14(2):285-95. doi: 10.1007/s11136-004-0705-2.

13. Guyatt GH, Walter S, Norman G. Measuring change over time: assessing the usefulness of evaluative instruments. J Chronic Dis.1987;40 (2): 171-8.DOI: 10.1016/0021-9681(87)90069-5

14. Xu QA, Zhang CM, Wan CH, Yang Z, Xu CZ, Chen Y. Computer simulation of MCID calculation method for minimum clinical significance difference. China Health Statistics.2017;34 (5): 785-7.

15. Jaeschke R, Singer J, Guyatt GH. Measurement of health status. Ascertaining the minimal clinically important difference. Control Clin Trials.1989;10 (4): 407-415.DOI: 10.1016/0197-2456(89)90005-6

16. Osoba D, Rodrigues G, Myles J, Zee B, Pater J. Interpreting the significance of changes in healthrelated quality-of-life scores. J Clin Oncol.1998;16 (1): 139-144.DOI: 10.1200/JC0.1998.16.1.139

17. Guyatt GH, Osoba D, Wu AW, Wyrwich KW, Norman GR; Clinical Significance Consensus Meeting Group. Methods to explain the clinical significance of health status measures. Mayo Clin Proc.2002;77 (4): 371-383.DOI: 10.4065/77.4.371 
18. Schünemann HJ, Guyatt GH. Goodbye (M) CID! Hello MID, where do you come from? (Commentary). Health Serv Res.2005;40 (2): 593-597.DOI: 10.1111/j.1475-6773.2005.00374.x

19. Wan $\mathrm{CH}, \mathrm{Yu} \mathrm{YL}$, Tan JF, Meng Q,Huang XP. Introduction to study of quality of life. Beijing: Science Press.2016;82-84.

20. Hu GQ, Huang QF, Huang ZN, SUN ZQ.A method for determining the smallest clinically significant change in clinical research. Journal of Central South University (Health Sciences).2009;34 (11): 1058-62.

21. Sun H, Xie Y, Li JS. Application of the minimum clinically important difference in COPD quality of life assessment tool.China General Practice.2015;18 (23): 2826-28.

22. Yang Z,Qi YB,Wan $\mathrm{CH}, \mathrm{Li} X \mathrm{XM}, \mathrm{Xu} \mathrm{CZ}$,Zhang $\mathrm{XQ}$,Zhao $\mathrm{ZH}, \mathrm{Li} \mathrm{H}$. Further analysis of the common module item response theory of the chronic disease patient quality of life scale system. Chinese General Practice,2012;15 (22): 2544-47.

23. Angst $F$, Aeschlimann A, Angst J. The minimal clinically important difference raised the significance of outcome effects above the statistical level, with methodological implications for future studies. J Clin Epidemiol.2017;82: 128-136.DOI: 10.1016/j.jclinepi.2016.11.016

24. Hanley JA,mcneil BJ. The meaning and use of the area under a receiver operating characteristic(Roc) curv. radiology, 1982,143(1):29-36.

25. Liu X,Xie T, Chen MY,Wan CH. Analysis of the current quality of life and influencing factors of patients with systemic lupus erythematosus in Zhanjiang area. Nursing Practice and Research,2017;14(08):1-4.

26. Chen WZ, Pan XP, Song XB, Ni ZX. Selection of the best working point in ROC curve .China Health Statistics.2006;(02): 157-8.

27. Song HL, He J, Yu HT, Li L. Evaluation and comparison of two related diagnostic tests using the area under the ROC curve. Journal of Second Military Medical University.2006; (05): 562-3.

28. J J Wu, C Lin, L Sun, O Goldblum, A Zbrozek, R Burge, M Augustin, S R Feldman. Minimal clinically important difference (MCID) for work productivity and activity impairment (WPAI) questionnaire in psoriasis patients. J Eur Acad Dermatol Venereol.2019;33(2):318-324.doi: 10.1111/jdv.15098.

29. Revicki D, Hays RD, Cella D, Sloan J. Recommended methods for determining responsiveness and minimally important differences for patient- reported outcomes. J Clin Epidemiol.2008;61 (2): 1029.DOI: 10.1016/j.jclinepi.2007.03.012

30. Turner D, Schünemann HJ, Griffith LE, Beaton DE, Griffiths AM, Critch JN,et al. Using the entire cohort in the receiver operating characteristic analysis maximizes precision of the minimal important difference. J Clin Epidemiol.2009;62 (4): 374-379.DOI: 10.1016/j.jclinepi.2008.07.009

\section{Figures}




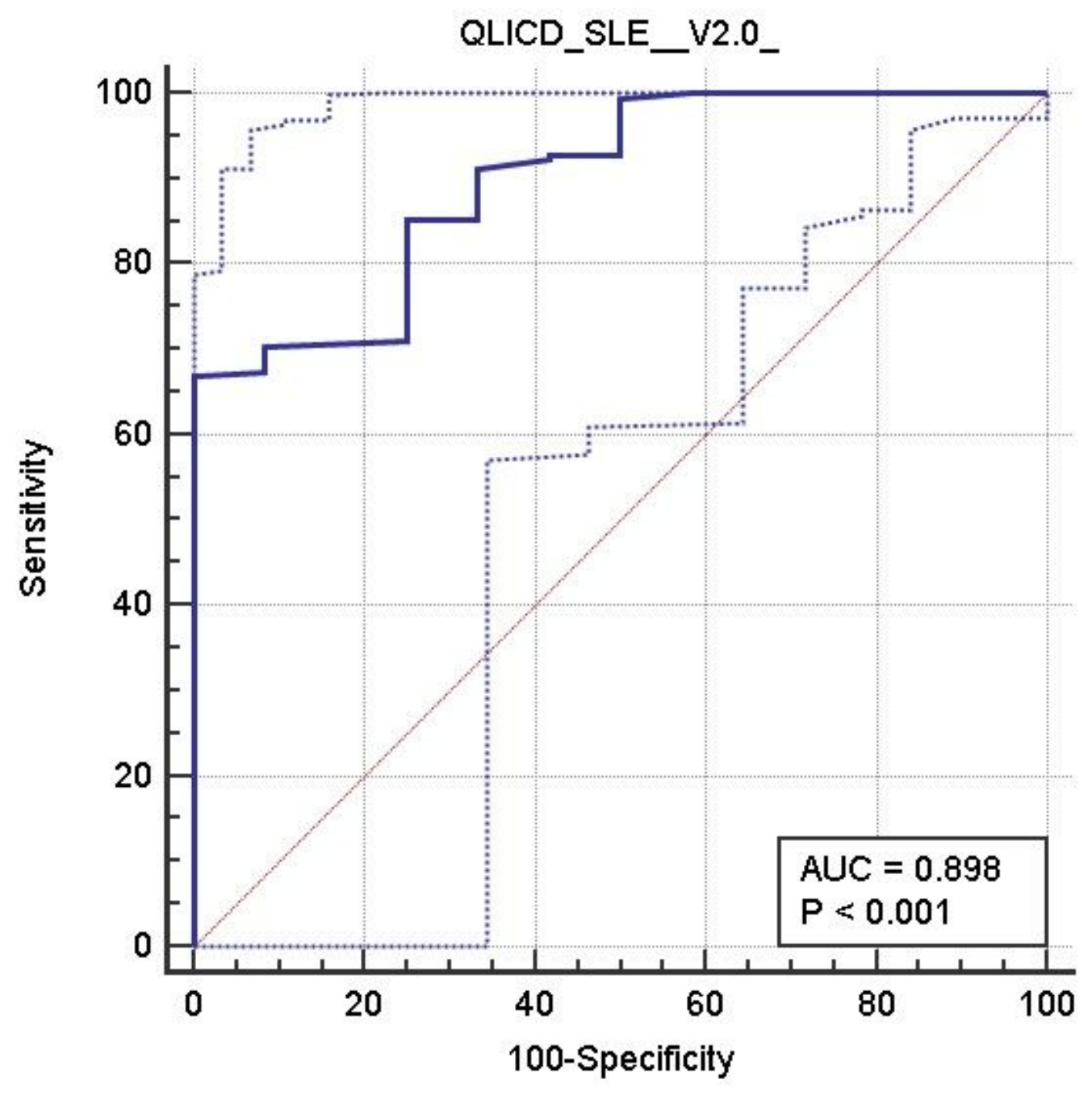

Figure 1

ROC graph of the QLICD-SLE (The sensitivity VS 100-specificity of the QLICD-SLE) Blue solid line: Fitted curve Blue dotted line: 95\% confidence interval of ROC curve Red solid line: Random line 


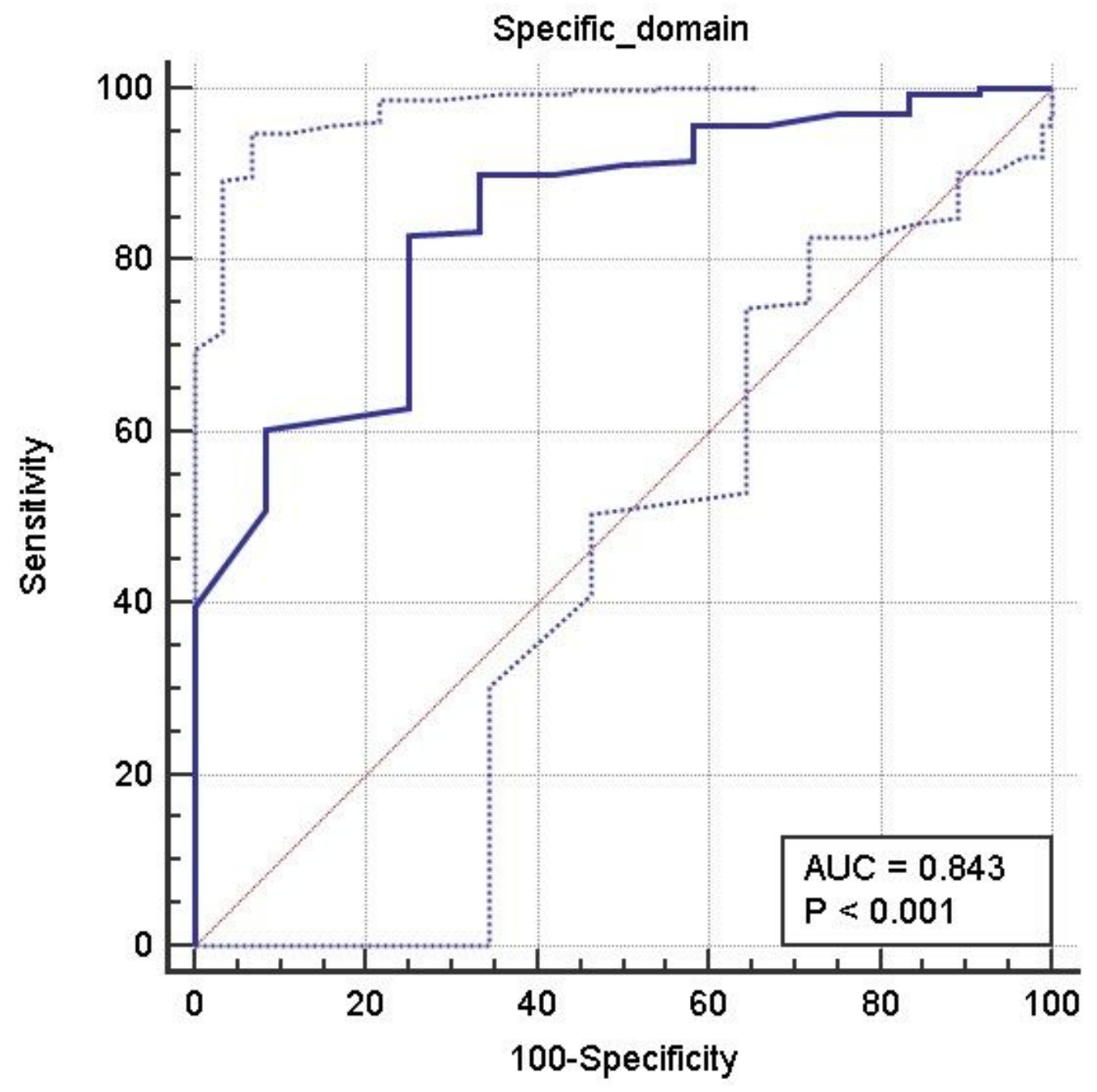

Figure 2

ROC graph of the Specific domain (The sensitivity VS 100-specificity of the specific domain of the QLICDSLE) Blue solid line: Fitted curve Blue dotted line: 95\% confidence interval of ROC curve Red solid line: Random line 